LBNL-42952

UCB-PTH-99/06

hep-ph/9903319

\title{
Late Inflation and the Moduli Problem of Sub-Millimeter Dimensions
}

\author{
Csaba Csáki周, Michael Graesser|, and John Terning \\ Theoretical Physics Group \\ Ernest Orlando Lawrence Berkeley National Laboratory \\ University of California, Berkeley, California 94720 \\ Department of Physics \\ University of California, Berkeley, California 94720 \\ ccsaki@lbl.gov, mlgraesser@lbl.gov, terning@alvin.lbl.gov
}

\begin{abstract}
We consider a recent model with sub-millimeter sized extra dimensions, where the field that determines the size of the extra dimensions (the radion) also acts as an inflaton. The radion is also a stable modulus, and its coherent oscillations can potentially overclose the Universe. It has been suggested that a second round of late inflation can solve this problem, however we find that this scenario does not allow for sufficient reheating of the Universe.
\end{abstract}

${ }^{*}$ Research fellow, Miller Institute for Basic Research in Science.

${ }^{\dagger}$ Supported by the Natural Sciences and Research Council of Canada. 
Recently a remarkable proposal has been made by Arkani-Hamed et. al. [1], suggesting that the fundamental Planck scale could be at the TeV scale provided that there are compact (sub-millimeter sized) extra dimensions that gravitational fields can propagate in. The radius of the extra dimensions then acts as a light (mass of order $10^{-3} \mathrm{eV}$ to order $\mathrm{MeV}$ ) dynamical field that is referred to as the radion [2]. A very attractive scenario [3] was then proposed in which the radion field itself can act as an inflaton in the early Universe, i.e. its vacuum energy density can dominate the energy density of the Universe and cause the three large spatial dimensions to expand exponentially in time. After this inflationary episode the Universe is reheated to a temperature of $10-100 \mathrm{MeV}$ with radiation being the dominant form of energy. The remaining energy density stored in the coherent oscillations of the radion is severely constrained so that it does not overclose the Universe. Thus the radion presents an example of a cosmological moduli problem, which is referred to as the radion problem in Refs. [2, 3]. In Ref. [3] it was proposed that a second round of inflation (a late inflation) with 5 to 6 e-foldings could sufficiently damp the radion oscillations. Here we will examine this proposal in some detail. We find that this late inflation generically shifts the minimum of the potential for the small radius, and that this shift is reliably calculable in these models. In order to achieve sufficient damping of the radion, the inflaton mass has to be extremely small. Even if such a light inflaton existed, it cannot reheat the Universe enough to allow for standard big-bang nucleosynthesis (BBN) to occur at temperatures around $1 \mathrm{MeV}$. Thus we argue that late inflation is an unlikely solution to the radion problem, which remains as one of the most severe problems for models with sub-millimeter dimensions. Needless to say, this result does not exclude the appealing framework of Ref. [3], but rather reiterates the difficulty of the moduli problem.

First we briefly review the effective Lagrangian approach of [2] to the equations of motion for the expanding universe. These equations are obtained by assuming that the metric of the $4+n$ dimensional spacetime is given by

$$
g_{\mu \nu}=\left(\begin{array}{ccc}
1 & & \\
& -R(t)^{2} g_{I J} & \\
& & -r(t)^{2} g_{i j}
\end{array}\right),
$$

where $R(t)$ is the time dependent scale factor of the large 4 dimensional space-time, $r(t)$ is the scale factor of the $n$ extra dimensions, while the $g_{I J}$ and $g_{i j}$ are flat metrics in 3 and $n$ dimensions respectively. The 3 large dimensions can be viewed as a "brane" or wall in the $4+n$ dimensional space-time. The effective Lagrangian for the system is obtained by plugging the background metric (11) into the action

$$
S=-\int d^{4+n} x \sqrt{-g}\left(M_{*}^{n+2} \mathcal{R}-\mathcal{L}\right),
$$

where $g=\operatorname{det} g_{\mu \nu}, \mathcal{R}$ is the curvature scalar, $M_{*}$ is the fundamental scale of the theory $\left(M_{*} \approx 1 \mathrm{TeV}\right)$, and $\mathcal{L}$ is the Lagrangian density which includes matter fields and cosmological constants both in the bulk and on the wall. By performing the integrals over the 
spatial coordinates, and integrating by parts to eliminate second time derivatives, an action dependent on $R, r$, and single time derivatives is obtained. The resulting Lagrangian for $R(t), r(t)$ is then given by [2]

$$
L_{\text {eff }}=-M_{*}^{n+2} R^{3} r^{n}\left(6\left(\frac{\dot{R}}{R}\right)^{2}+n(n-1)\left(\frac{\dot{r}}{r}\right)^{2}+6 n\left(\frac{\dot{r} \dot{R}}{r R}\right)\right)-V_{\text {eff }}(r, R) .
$$

Here $V_{\text {eff }}$ includes the potential which stabilizes the radius of the extra dimensions to a value $r_{0}$ and the potential of the matter fields on the wall which in our case will lead to the late inflation under discussion:

$$
V_{\text {eff }}(r, R)=R^{3}\left(V_{b u l k}(r)+V_{\text {wall }}(R)\right)
$$

Since we are interested in the epoch after the initial inflation, at times when the radion is already stabilized close to its actual minimum $r_{0}$, a good approximation for $V_{\text {bulk }}$ is to take it to be quadratic. The mass of the radion has been calculated in Ref. [2] to be

$$
m_{n}^{2}=\frac{V_{b u l k}^{\prime \prime}\left(r_{0}\right)}{n(n+2) M_{*}^{n+2} r_{0}^{n-2}}
$$

where $m_{n}$ is the radion mass, which was found to be [2] between the $n$ independent lower bound of $10^{-3} \mathrm{eV}$, and an upper bound of $10^{-2} \mathrm{eV}$ for $n=2$ or an upper bound of $20 \mathrm{MeV}$ for $n=6$. Thus our approximation for $V_{\text {bulk }}$ is

$$
V_{\text {bulk }}(r)=\frac{1}{2} n(n+2) m_{n}^{2} M_{*}^{n+2} r_{0}^{n-2}\left(r-r_{0}\right)^{2} .
$$

Introducing dimensionless variables (which we will use from here on) $r \rightarrow r_{0} r, R \rightarrow r_{0} R$, the equations of motion for the new variables obtained from the effective Lagrangian (3) are:

$$
\begin{aligned}
0= & -(n-1) r^{n-2}\left(6 \frac{\dot{r} \dot{R}}{R}+2 \ddot{r}+(n-2) \frac{\dot{r}^{2}}{r}\right)-6 r^{n-1}\left(\frac{\dot{R}^{2}}{R^{2}}+\frac{\ddot{R}}{R}\right)+(n+2) m_{n}^{2}(r-1) \\
0= & -3 n r^{n-1}\left((n-1) \frac{\dot{r}^{2}}{r}+2 \ddot{r}\right)-12 n r^{n-1} \frac{\dot{r} \dot{R}}{R}-6 r^{n}\left(\left(\frac{\dot{R}}{R}\right)^{2}+2 \frac{\ddot{R}}{R}\right) \\
& +3 m_{n}^{2} \frac{n(n+2)}{2}(r-1)^{2}+\frac{1}{M_{\mathrm{Pl}}^{2}}\left(3 V_{\text {wall }}+R V_{\text {wall }}^{\prime}\right),
\end{aligned}
$$

where the reduced Planck scale is given by $M_{\mathrm{Pl}}^{2} \equiv M_{*}^{n+2} r_{0}^{n}=\left(2 \times 10^{18} \mathrm{GeV}\right)^{2}$.

Next we assume that the theory contains an inflaton field which produces a second period of inflation (a late inflation) after the initial inflation due to the radion, and explore 
the consequences. We assume that the vacuum energy of the Universe is dominated for a brief time by the vacuum energy of this field, which we parameterize as:

$$
V_{\text {wall }} \approx V_{I}=\lambda^{2} M_{\mathrm{Pl}}^{2}+\cdots
$$

where the ellipsis indicates other field dependent terms. This vacuum energy will force the scale factor of the large dimensions, $R$, to grow as

$$
R \propto e^{H t}, \quad H \approx \frac{\lambda}{\sqrt{6}} .
$$

During this inflationary period the oscillations in the radion are rapidly damped. One can easily see that the coupling of the radion field to the scale factor of the large dimensions introduces a shift in the effective potential experienced by the oscillating radion field. From Eq. (7) we can read off the derivative of this potential:

$$
V_{\text {eff }, \text { bulk }}^{\prime}(r)=V_{\text {bulk }}^{\prime}(r)-6 n r^{n-1}\left(\frac{\dot{R}^{2}}{R^{2}}+\frac{\ddot{R}}{R}\right) .
$$

It is this shift in the minimum of the potential [5] that will provide the crucial constraint on these late inflation models. The effect of inflation shifting the effective potential of a modulus has been known for a long time, and in fact can be viewed as the real origin of the moduli problem [5]. However, in generic models the size of the shift of the modulus usually depends on unknown physics (for example on higher order Kähler potential couplings in the case of supersymmetric theories), and thus cannot be reliably estimated. In this example however the shift is just given by solving Eq. (7).

In order to obtain a model that gives the appropriate conditions to form the observed Universe, the energy density in the radion oscillations must be very small. If, however, the late inflation damps the oscillations around a minimum that is far from the true minimum after inflation, then the radion will again begin to oscillate around its true minimum once inflation has ended. Thus we find that this shift in the minimum during inflation must be quite small; this requires that $\lambda$ be much smaller than $m$. This can be seen by looking for the steady-state inflationary solution, taking $r$ to be a constant $r=r_{I}$, and $R$ to grow exponentially, as in Eq. (9). These forms exactly solve the equations of motion and give the minimum during inflation as

$$
r_{I}=\frac{(2 n-1) m_{n} \pm \sqrt{m_{n}^{2}-8\left(\frac{n-1}{n+2}\right) \lambda^{2}}}{2 m_{n}(n-1)} .
$$

The negative sign gives the solution that approaches the true minimum as $\lambda \rightarrow 0$. For small $\lambda$ this can be approximated as

$$
r_{I} \approx 1+\frac{2 \lambda^{2}}{(n+2) m_{n}^{2}}
$$

\footnotetext{
${ }^{*}$ For $\lambda \gg m_{n}$, Eq. (11] has no real solutions, and no damping of the moduli oscillations occurs [5].
} 
This result can also be easily obtained from Eq. (10) by setting $V_{\text {eff }}^{\prime}=0$ and linearizing in $\lambda^{2} / m^{2}$.

The energy density stored in the radion field at the end of the second stage of inflation is given by Eq. (6)

$$
V=\frac{1}{2} n(n+2) m_{n}^{2} M_{\mathrm{Pl}}^{2}\left(r_{I}-1\right)^{2} .
$$

In order for this energy density to not overclose the Universe today (i.e. to not reintroduce the radion problem), it is bounded by

$$
\frac{V}{T_{R H}^{3}}<\frac{3}{2} \times 10^{-9} \mathrm{GeV}
$$

where $T_{R H}$ is the reheat temperature of the Universe at the end of the late inflationary period, and we have set the current Hubble parameter to $H_{0}=50 \mathrm{~km} \mathrm{~s}^{-1} \mathrm{Mpc}^{-1}$. This in turn implies an upper bound on the shift in $r$ at the end of the late inflation:

$$
\left(r_{I}-1\right)<3 \times 10^{-14} \frac{1}{\sqrt{n(n+2)}}\left(\frac{10^{-3} \mathrm{eV}}{m_{n}}\right)\left(\frac{T_{R H}}{10 \mathrm{MeV}}\right)^{3 / 2} .
$$

A model independent, and $n$ independent, upper limit is obtained by inserting $n=2$ and the lower bound $m_{n}>10^{-3} \mathrm{eV}$ on the radion mass, which is determined from short-distance force experiments [4]. This gives

$$
\left(r_{I}-1\right)<10^{-14}\left(\frac{T_{R H}}{10 \mathrm{MeV}}\right)^{3 / 2} .
$$

We note that the actual constraint on $r_{I}$ from Eq. (15), for a given $m_{n}$ and $n$, can be much stronger. Next, using Eq. (12), and the upper limit on $r_{I}$ given in Eq. (15), an upper bound on the inflationary scale $\lambda$ is:

$$
\begin{aligned}
& \lambda<4 \times 10^{-16} \mathrm{MeV}\left(\frac{T_{R H}}{10 \mathrm{MeV}}\right)^{3 / 4}\left(\frac{m_{n=2}}{10^{-2} \mathrm{eV}}\right)^{1 / 2}, n=2, \\
& \lambda<8 \times 10^{-12} \mathrm{MeV}\left(\frac{T_{R H}}{10 \mathrm{MeV}}\right)^{3 / 4}\left(\frac{m_{n=6}}{5 \mathrm{MeV}}\right)^{1 / 2}, n=6 .
\end{aligned}
$$

Provided that the shift in the minimum of the potential is sufficiently small, then 5 to 6 e-foldings of inflation are required in order to sufficiently damp the radion oscillations. The number of e-foldings is given by [7]

$$
N=\int H d t=\int d \phi \frac{3 H^{2}}{V_{I}^{\prime}} \approx \frac{\Delta \phi \lambda^{2}}{2 V_{I}^{\prime}}
$$


where $\phi$ is the inflaton field, and $\Delta \phi$ is the distance in field space that it travels during the course of inflation. This equation simply constrains the inflaton potential to be sufficiently flat during inflation. In addition the slow-roll condition requires that $\left|V_{I}^{\prime \prime}\right| \ll 9 H^{2}$. For a natural potential (where there are no fine-tuned cancelations between terms) each term in the potential should have sufficiently small derivatives in order for slow-roll inflation to occur. These constraints imply a bound on the mass of the inflaton field:

$$
m_{I}<\lambda \text {. }
$$

In order to avoid significant cosmological difficulties, the reheat temperature $T_{R H}$ must be less than the "normalcy" temperature $T_{*}$ [6] below which the $4 \mathrm{D}$ Universe is radiation dominated with the bulk essentially empty of energy. Processes such as $\gamma \gamma \rightarrow$ bulk graviton occurring in the early Universe can dump too much energy into the bulk if the temperature is above $T_{*}$. The over-production of bulk gravitons, for example, can significantly affect the expansion rate of the Universe during BBN and also overclose the Universe. Furthermore, the late decay of Kaluza-Klein gravitons to two photons can produce spikes in the background photon spectrum and is a very significant cosmological constraint. Since the late photon constraint may be avoided in models where the bulk is populated with many branes, we instead use the (weaker) constraint that is obtained from requiring that the energy density in the bulk gravitons is less than about a tenth of the energy in radiation during BBN [6]. This leads to the constraint: $T_{R H}<3 \times 10^{-6} M_{*}$ for $n=2$, and $T_{R H}<2 \times 10^{-3} M_{*}$ for $n=6$. Inserting these upper bounds on $T_{R H}$ into Eq. (17) and Eq. (18) gives an upper bound on the mass of the inflaton in terms of $M_{*}$ :

$$
\begin{aligned}
& m_{I}<2 \times 10^{-16} \mathrm{MeV}\left(\frac{m_{n=2}}{10^{-2} \mathrm{eV}}\right)^{1 / 2}\left(\frac{M_{*}}{1 \mathrm{TeV}}\right)^{3 / 4}, \quad n=2, \\
& m_{I}<4 \times 10^{-10} \mathrm{MeV}\left(\frac{m_{n=6}}{5 \mathrm{MeV}}\right)^{1 / 2}\left(\frac{M_{*}}{1 \mathrm{TeV}}\right)^{3 / 4}, \quad n=6 .
\end{aligned}
$$

Since the initial round of inflation in this model is thought to reheat the Universe to a temperature around $10-200 \mathrm{MeV}, 5$ or 6 e-foldings will result in a Universe too cold for the BBN scenario which requires a radiation dominated Universe at temperatures of a few $\mathrm{MeV}$. Therefore the Universe must be reheated again after the second inflationary period, and the inflaton must therefore decay.

This can be made more precise. Denote by $T_{1}$ and $V_{1}$ the temperature of the radiation and the energy density in radion oscillations, respectively, at the onset of the late (second) inflationary phase. Then the temperature at the end of inflation, but before reheating, is $T_{2}=e^{-N} T_{1}$. The energy in radion oscillations at the end of inflation is $V=e^{-3 N} V_{1}+V_{\text {shift }}>$ $e^{-3 N} V_{1}$. Here $V_{\text {shift }}$ is the energy density due to the shift in the minimum of the potential during the late inflation. Then the overclosure constraint implies

$$
\frac{3}{2} \times 10^{-9} \mathrm{GeV}>\frac{V}{T_{R H}^{3}}>\left(\frac{T_{2}}{T_{R H}}\right)^{3} \frac{V_{1}}{T_{1}^{3}}=\left(\frac{T_{2}}{T_{R H}}\right)^{3} \frac{V_{i}}{T_{i}^{3}} .
$$


In the last equality we have used $V / T^{3}=$ constant to express the result in terms of $T_{i}$ and $V_{i}$, the values of $T$ and $V$, respectively, at the end of the reheating following the first inflationary phase, rather than their values at the start of the second inflationary phase. If there is a moduli problem then $V_{i} \sim T_{i}^{4}$. In fact this condition holds at the end of the first reheating in the model of Ref. [3]. Assuming that no secondary reheating is required, then $T_{R H}=T_{2}<T_{i}$. Inserting this and $V_{i} \sim T_{i}^{4}$ into Eq. (23) leads to an unacceptable conclusion, namely $T_{i} \ll \mathcal{O}(\mathrm{MeV})$. Alternatively, in the framework of Ref. [3], the temperature of the Universe at the end of the first reheating (but before the start of the late inflationary phase) is $T_{i} \sim 2-200 \mathrm{MeV}$, for $n=2$ to $n=5$. Inserting these values of $T_{i}$ into the above formula, Eq. (23), implies that $T_{2}$ is a tiny fraction of $T_{R H}$ :

$$
T_{2}<2 \times 10^{-2} T_{R H}
$$

Therefore a second reheating of the Universe must occur.

In order to reheat the Universe, the inflaton must decay to particles that have standard model interactions. The only particles that are sufficiently light (given the current experimental data) are photons and neutrinos. We will assume that the inflaton is neutral and decays directly to photons rather than through an additional intermediary that subsequently decays to photons. Thus the simplest possibility is that the inflaton decays to two photons through a dimension 5 operator, $\phi F_{\mu \nu} F^{\mu \nu}$, suppressed by the fundamental scale $M_{*} \approx 1$ TeV. Given the limit in Eq. (21) and Eq. (22), it not even clear that the decay to massive neutrinos is kinematically allowed. If it is allowed, then a direct decay to two neutrinos, by gauge-invariance, must also proceed through a dimension 5 operator. In any case, the decay rate is comparable to (or smaller than) the two photon decay. A conservative estimate of the decay width is then

$$
\Gamma \approx \frac{m_{I}^{3}}{M_{*}^{2}} .
$$

This leads to a very small reheat temperature [7]:

$$
T_{R H} \approx 1.2 g_{*}^{-1 / 4} \sqrt{\Gamma M_{\mathrm{Pl}}}
$$

where $g_{*}$ counts the number of degrees of freedom that are in equilibrium at a given temperature $\left(g_{*} \approx 10\right.$ at $\mathrm{MeV}$ temperatures in the Standard Model). Using the upper limit to $m_{I}$, Eq. (21) and Eq. (22), we find a reheat temperature of

$$
\begin{aligned}
& T_{R H}<8 \times 10^{-20} \mathrm{MeV}\left(\frac{M_{*}}{1 \mathrm{TeV}}\right)^{1 / 8}\left(\frac{m_{n=2}}{10^{-2} \mathrm{eV}}\right)^{3 / 4}, n=2 \\
& T_{R H}<3 \times 10^{-10} \mathrm{MeV}\left(\frac{M_{*}}{1 \mathrm{TeV}}\right)^{1 / 8}\left(\frac{m_{n=6}}{5 \mathrm{MeV}}\right)^{3 / 4}, n=6 .
\end{aligned}
$$


The reheat temperatures given by Eqs. (27) and (28) are far too small for successful BBN.

To summarize we have found that the inflaton necessary to damp the radion must be remarkably light, and that such light inflatons cannot successfully reheat the Universe to BBN temperatures. Of course our conclusions can be avoided with a sufficient amount of fine-tuning. For example if the inflaton potential is extremely fine-tuned (which could perhaps arise from some unknown physics), then the bound (20) on the inflaton mass can be avoided. One can also imagine adding additional interactions between the radion and the inflaton which are fine-tuned to cancel the shift in the minimum of the radion potential (in this case the inflaton potential would also have to be fine-tuned so as to remain sufficiently flat for inflation to occur). Finally the fundamental scale $M_{*}$ could be made much larger than $1 \mathrm{TeV}$ so that the bound on the radion and inflaton masses could be relaxed, but this results in a hierarchy between the weak scale and the fundamental scale, and a corresponding fine-tuning of the Higgs mass. This is very unappealing, since the absence of a fine-tuned Higgs mass is one of the major motivations for the sub-millimeter extra dimension scenario. Thus, in the absence of fine-tuning, the moduli problem of sub-millimeter extra dimensions remains a difficult problem that requires a more interesting solution than a standard late inflation.

\section{Acknowledgements}

We are grateful to Nima Arkani-Hamed, Chris Kolda, and Hitoshi Murayama for useful discussions. This work was supported in part by the U.S. Department of Energy under Contract DE-AC03-76SF00098 and in part by the National Science Foundation under grant PHY-95-14797. C.C. is a research fellow of the Miller Institute for Basic Research in Science. M. G. is supported by the Natural Sciences and Engineering Research Council of Canada.

\section{References}

[1] N. Arkani-Hamed, S. Dimopoulos, and G. Dvali, Phys. Lett. 429B, 263 (1998), hepph/9803315; I. Antoniadis, N. Arkani-Hamed, S. Dimopoulos, and G. Dvali, Phys. Lett. 436B, 257 (1998), hep-ph/9804398.

[2] N. Arkani-Hamed, S. Dimopoulos, and J. March-Russell, hep-th/9809124.

[3] N. Arkani-Hamed, S. Dimopoulos, N. Kaloper, J. March-Russell, hep-ph/9903224.

[4] R. Sundrum, hep-ph/9805471, hep-ph/9807348.

[5] A. Goncharov, A. Linde, M. Vysotsky, Phys. Lett. 147B, 279 (1984); M. Dine, L. Randall, S. Thomas, Phys. Rev. Lett. 75, 398 (1995), hep-ph/9503303; L. Randall, S. 
Thomas, Nucl. Phys. B449, 229 (1995), hep-ph/9407248; A. Linde, Phys. Rev. D53, 4129 (1996), hep-th/9601083.

[6] N. Arkani-Hamed, S. Dimopoulos, and G. Dvali, hep-ph/9807344.

[7] E. Kolb and M. Turner, "The Early Universe," (Addison-Wesley, 1990). 